\title{
IRF1 association with tumor immune microenvironment and use as a diagnostic biomarker for colorectal cancer recurrence
}

\author{
YANFANG WU ${ }^{1}$, SHUJU ZHANG ${ }^{2}$ and JUN YAN ${ }^{3}$ \\ ${ }^{1}$ Department of Gastroenterology, The Fourth People's Hospital of Shaanxi, Xi'an, Shanxi 710032; \\ ${ }^{2}$ Hunan Children's Research Institute, Hunan Children's Hospital, University of South China, Changsha, Hunan 410007; \\ ${ }^{3}$ Center of Hepatobiliary Pancreatic Disease, Beijing Tsinghua Changgung Hospital, Beijing 102218, P.R. China
}

Received June 19, 2019; Accepted November 27, 2019

DOI: $10.3892 / \mathrm{ol} .2020 .11289$

\begin{abstract}
Colorectal cancer (CRC) is considered to be one of the most lethal cancer types globally, and its recurrence is a major treatment challenge. Identifying the factors involved when determining the risk of CRC recurrence is required to improve personalized therapy for patients with CRC. Based on the GSE39582 dataset, the present study demonstrated that a higher ratio of M1 macrophages and activated memory $\mathrm{CD}^{+} \mathrm{T}$ cells indicated a better recurrence-free survival (RFS) time for CRC, using CIBERSORT and Pearson's correlation analysis. Through weighted correlation network analysis (WGCNA), an immune-associated module was identified that was significantly positively correlated with the ratio of M1 macrophages and activated memory $\mathrm{CD}^{+} \mathrm{T}$ cells. In this module, using WGCNA and a protein-protein interaction network, interferon regulatory factor 1 (IRF1), chemokine ligand 5, ubiquitin/ISG15-conjugating enzyme E2 L6, guanylate binding protein 1 and interleukin 2 receptor subunit beta were identified as hub genes. Among these genes, univariate Cox and multivariate Cox analysis revealed that IRF1 may be a potential diagnostic biomarker for RFS in patients with CRC. This was further validated using The Cancer Genome Atlas data. Gene set enrichment analysis demonstrated that IRF1 influenced the genes and pathways that are associated
\end{abstract}

Correspondence to: Professor Yanfang Wu, Department of Gastroenterology, The Fourth People's Hospital of Shaanxi, 512 Xianningdong Road, Xi'an, Shanxi 710032, P.R. China

E-mail: wuyanfang1121@sina.com

Abbreviations: CRC, colorectal cancer; WGCNA, weighted gene co-expression network analysis; GO, Gene Ontology; KEGG, Kyoto Encyclopedia of Genes and Genomes; COAD, Colon Adenocarcinoma; GEO, Gene Expression Omnibus; BP, biological process; FDR, false discovery rate; TCGA, The Cancer Genome Atlas; TNM, Tumor-Node-Metastasis; RFS, recurrence-free survival; TME, tumor microenvironment

Key words: colorectal cancer recurrence, tumor microenvironment, M1 macrophages, activated memory $\mathrm{CD}^{+} \mathrm{T}$ cells, interferon regulatory factor 1, DNA methylation with immune cell recruitment and activation. Additionally, the DNA methylation of cg27587780 and cg15375424 CpG sites in the IRF1 gene region was indicated to be negatively correlated with IRF1 mRNA expression and positively correlated with the recurrence of CRC. Collectively, the results of the present study demonstrated that IRF1 may be a potential diagnostic biomarker for RFS in patients with CRC.

\section{Introduction}

Colorectal cancer (CRC) is one of the most common cancer types and the leading cause of cancer-associated mortality globally $(1,2)$. The current standard treatment for colon cancer is surgery combined with chemotherapy. However, a proportion of patients still suffer from local recurrence and remote metastasis following surgery (3). Furthermore, patients with similar clinical or pathological conditions may exhibit unpredictable and diversified clinical outcomes, even when treated in the same way. This phenomenon reveals the limitation of the classic tumor-node-metastasis (TNM) staging system (4). Reliable and robust molecular markers, in addition to the current clinical and pathological factors used for determining the risk of CRC recurrence, are required to improve personalized therapy for patients (5).

The development of bioinformatics and gene expression profiling technologies provides additional opportunities to characterize the molecular features of cancer. Gene-expression profiling has been used to develop genomic tests that may provide better predictions of clinical outcomes in combination with traditional clinicopathologic factors (6). Although a number of studies have used this method in CRC, to the best of our knowledge, this has not been applied clinically (7). Therefore, establishing a novel and more effective signature for assessing CRC recurrence is urgently required.

Previously, emerging evidence has suggested that tumor progression and recurrence are not only regulated by the abnormal gene expression of cancer cells but also by the tumor microenvironment (TME) factors, including the infiltration of a number of immune cell subsets (8). The expression of TME-associated genes, including chemokine and inflammatory factors, affects the infiltration of immune cells in TME, and is associated with the recurrence and survival of patients with cancer (9). 
In the present study, the association between immune cell subtypes in CRC tumor microenvironment and recurrence-free survival (RFS) time was evaluated. Subsequently, to explore the hub genes that are associated with the immune cell subtypes in the TME, weighted correlation network analysis, protein-protein interaction network, univariate Cox and multivariate Cox analyses were sequentially performed. Eventually, the influence of the degree of DNA methylation of hub genes-associated $\mathrm{CpG}$ sites on their expression was evaluated.

\section{Materials and methods}

Collection of gene expression datasets. GSE39582 were downloaded from the Gene Expression Omnibus (GEO) database (https://www.ncbi.nlm.nih.gov/geo/query/acc. cgi?acc=GSE39582) (10). GSE39582 includes 585 colorectal cancer samples and 19 non-tumoral tissue samples, which were from a French cohort (11). GSE71187 data were downloaded from the GEO database (https://www.ncbi.nlm.nih. gov/geo/query/acc.cgi?acc=GSE71187), which included a Chinese cohort with the mRNA expression profiles of 52 human biopsy samples of CRC, and included overall survival time information (12). The DNA methylation profile, RNA-seq raw counts and clinical information of 234 patients with CRC was retrieved from The Cancer Genome Atlas (TCGA) data portal using R package TCGAbiolinks (13).

Immune cell infiltration analysis. CIBERSORT (https://cibersort.stanford.edu/) (14) is an online analytical tool that is used to provide an estimation of the abundance of a number of immune cell sub-types in a mixed cell population using gene expression data. LM22 (downloaded from https://media.nature.com/original/nature-assets/nmeth/journal/v12/n5/extref/nmeth.3337-S2. xls) was used as the signature gene file for distinguishing 22 immune cell types in CIBERSORT $(14,15)$. The GSE39582 expression mixture file was uploaded to the CIBERSORT website. CIBERSORT was subsequently run online to calculate the ratio of the 22 immune cell subsets, due to the mRNA expression of immune signature genes in the cancer tissue of patients with CRC, and non-tumoral tissue samples. Permutations was set to 1,000, quantile normalization was used for GSE39582 gene chip data and disable quantile normalization was used for TCGA CRC RNA-seq data (14).

Recurrence-associated genes screening. The genes combined with recurrence clinical traits, which were significant in univariate Cox analysis, were identified as recurrence-associated genes based on GSE39582. R package survival (version 2.42-6; https://cran.r-project.org/web/packages/survival/index.html) was used for univariate Cox analysis (16). $\mathrm{P}<0.05$ was considered to indicate a statistically significant difference. Hazard ratios (HRs) were used to identify protective $(\mathrm{HR}<1)$ and risk genes $(\mathrm{HR}>1)(17)$.

Weighted correlation network analysis (WGCNA). The WGCNA package (version 1.63), in $\mathrm{R}$, was used to assess correlation patterns among genes, and identify modules of highly correlated genes (18). WGCNA was used in the present study to construct the co-expression network for the recurrence-associated genes that were identified using univariate Cox analysis, as described previously (17). $\beta$ was a soft-thresholding parameter that was able to emphasize strong correlations between genes and penalize weak correlations $(18,19)$ ). The power of $\beta=4$ (scale free $R^{2}=0.8$ ) was selected as the soft-threshold to ensure a scale-free network. A cut height of 0.25 and minimum size of 50 were used to identify modules. Pearson's correlation matrices were subsequently calculated between each module and immune cell subset.

Enrichment analysis. R package clusterProfiler (version 3.9.1; http://www.bioconductor.org/packages/release/bioc/html/clusterProfiler.html) was used for Gene Ontology (GO) Biological Process and Kyoto Encyclopedia of Genes and Genomes (KEGG) pathway enrichment analysis of the genes in modules that were identified using WGCNA (20). P<0.05 was considered to indicate a statistically significant difference.

Protein-protein interaction (PPI) network construction. STRING (https://string-db.org/) (21) was used to construct the PPI networks for distance-associated genes using the genes from modules identified by WGCNA. The PPI networks were then exported from STRING and imported into Cytoscape (22).

Hub gene identification. To identify the hub genes, all the listed genes were ranked based on their degrees calculated in the PPI network and co-expression network. The sum rank $\left(\mathrm{N}_{\text {sum rank }}=\mathrm{N}_{\text {ppi rank }}+\mathrm{N}_{\text {co-expression rank }}\right)$ was then used to identify hub genes.

Gene set enrichment analysis (GSEA). Patients in GSE39582 and TCGA Colon Adenocarcinoma (COAD) were divided into two groups (an IRF1 high-expression group and an IRF1 low-expression group) based on the median of IRF1 expression in each dataset. The median of IRF1 expression was of 11.6 and 7.23 for TCGA COAD and GSE39582 dataset, respectively. $\mathrm{R}$ package clusterProfiler was then used for GSEA to compare the different KEGG pathways between the two groups (23).

Methylation analysis. As the GSE39582 dataset lacked methylation data, the TCGA CRC Level 3 450K DNA methylation dataset was used for the further analysis of DNA methylation. The acquired methylated sites were annotated based on the GPL13534 (Illumina HumanMethylation450 BeadChip; Illumina, Inc.) annotation information. Pearson's correlation coefficient was used to evaluate the relevance between gene expression and the degree of methylated $\mathrm{CpG}$ sites (24). A Wilcoxon's test was used to select the differentially methylated $\mathrm{CpG}$ sites between the two groups (25). $\mathrm{P}<0.05$ was considered to indicate a statistically significant difference.

Statistical analysis. Statistical analysis was performed using $\mathrm{R}$ (version 3.5.3). The rank-sum t-test was used to test the differences between two groups. A one-way analysis of variance was used to test the differences among multiple groups, using the Tukey-honestly significant difference test as the post-hoc test (26). R package survival (version 2.42-6) was used for Kaplan-Meier survival analysis and the log-rank test. $\mathrm{R}$ package corrplot (version 0.84 ) was used to calculate the 
A

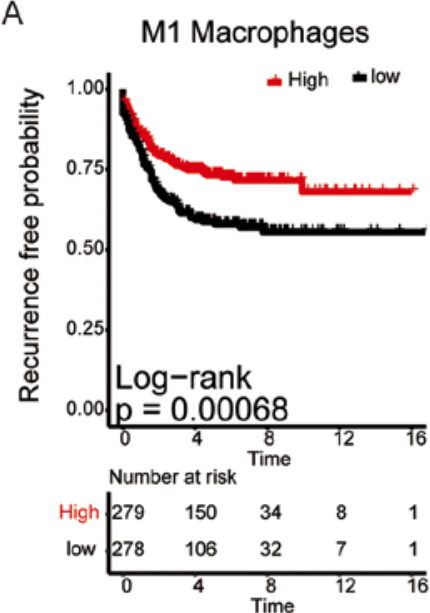

B

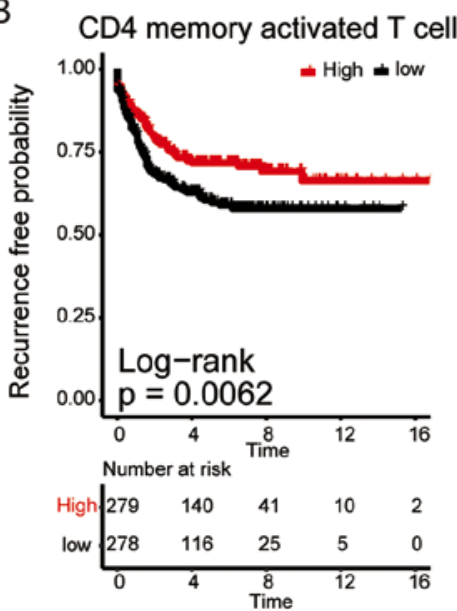

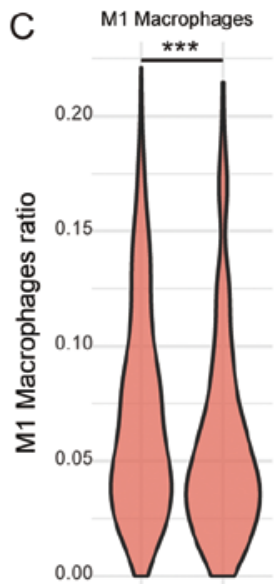

no recurrence recurrence

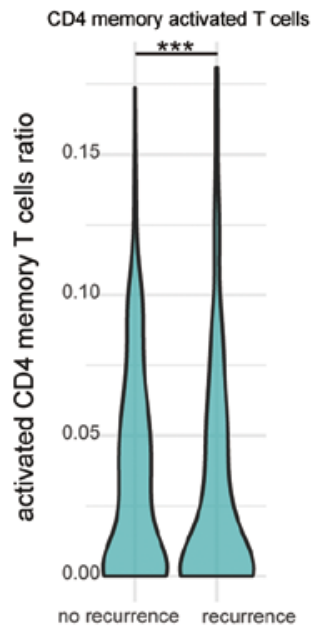

BRAF
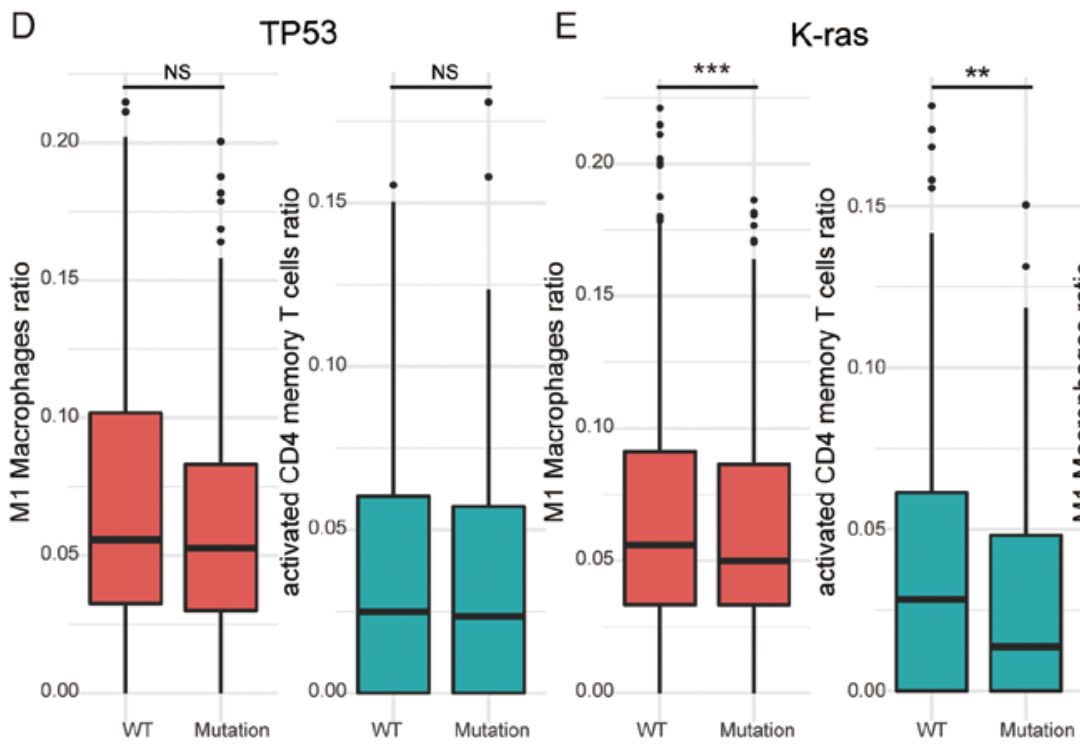

F

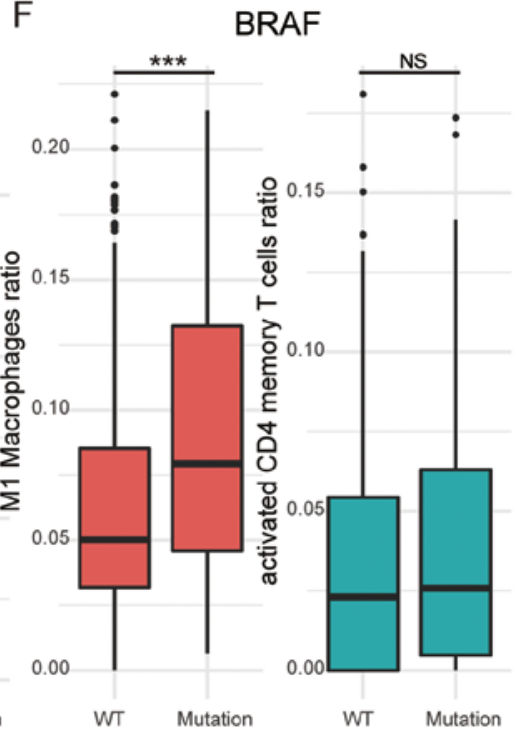

G $\quad$ M1 Macrophages in different TNM stage

CD4 memory activated T cells in different TNM stage
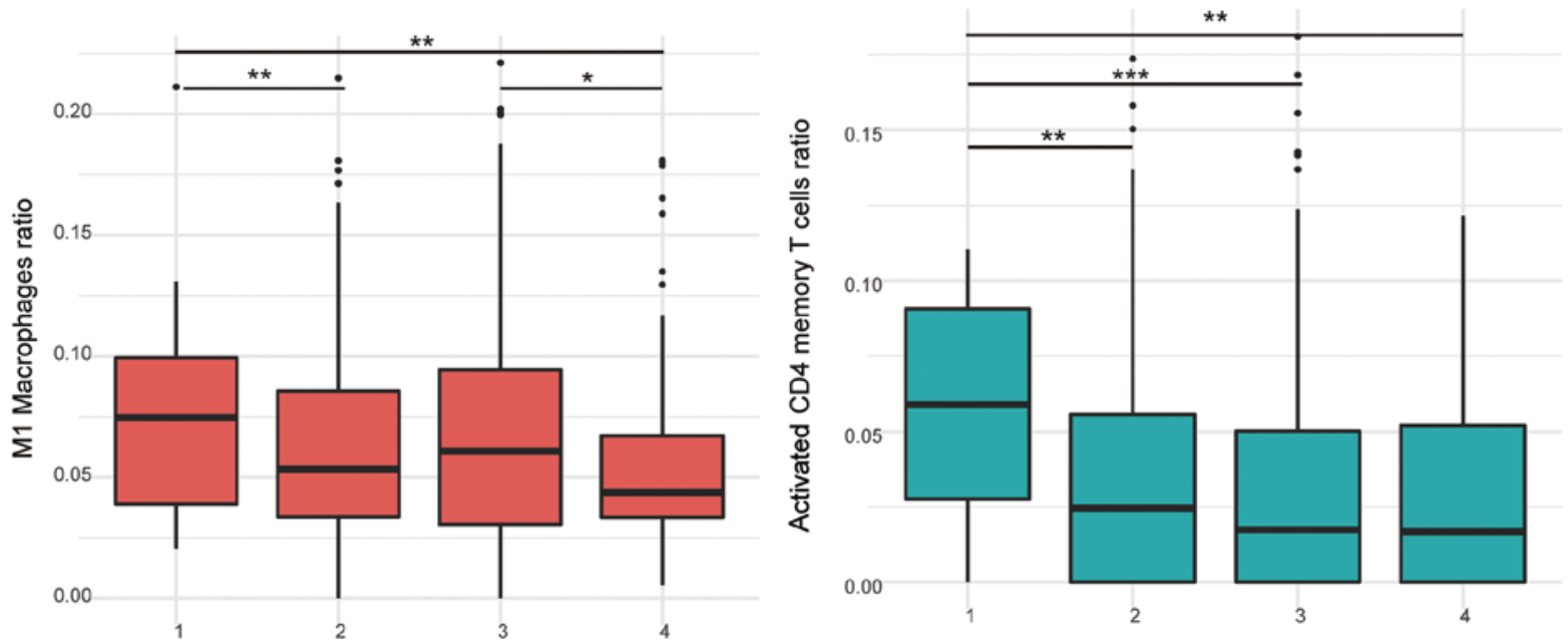

Figure 1. Function of immune cell infiltration in TME for recurrence. (A and B) Kaplan-Meier survival plots of RFS. According to the median infiltration level of (A) M1 macrophages and (B) activated $\mathrm{CD}^{+}$memory $\mathrm{T}$ cells, the patients were stratified into a high-level group and a low-level group. (C) A violin plot of the ratio of M1 macrophages and activated $\mathrm{CD} 4^{+}$memory T cells in no-recurrence/recurrence patients. (D-F) Box plots indicated the ratio of M1 macrophages and activated $\mathrm{CD}^{+}$memory T cells (green) in the cohorts of (D) wild-type or mutated TP53, (E) K-ras and (F) BRAF. (G) A box plot demonstrated the ratio of M1 macrophages (left) and activated $\mathrm{CD} 4^{+}$memory $\mathrm{T}$ cells (right) among different TNM stages. The vertical axes indicated the lower quartile and upper quartile of the ratio of M1 macrophages (red) or activated CD4 $4^{+}$memory $\mathrm{T}$ cells (green); and the line inside each box indicates the median ratio of M1 macrophages or activated $\mathrm{CD} 4{ }^{+}$memory $\mathrm{T}$ cells in each group $(\mathrm{D}-\mathrm{G}) .{ }^{*} \mathrm{P}<0.05,{ }^{* * *} \mathrm{P}<0.01$ and ${ }^{* * * *} \mathrm{P}<0.001$ with comparisons shown by lines. Log-rank test for $(\mathrm{A}) \mathrm{t}$-test for (B-F) and one-way analysis of variance followed by Tukey-honestly significant difference tests as post-hoc tests for (G). TME, tumor microenvironment; RFS, recurrence free survival; TNM, tumor-node-metastasis; BRAF, B-Raf proto-oncogene, serine/threonine kinase. 
A

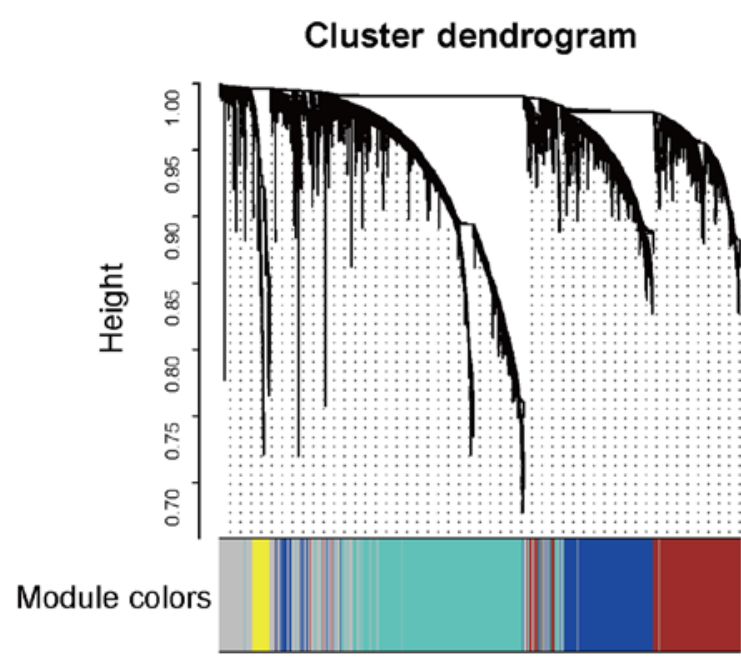

C

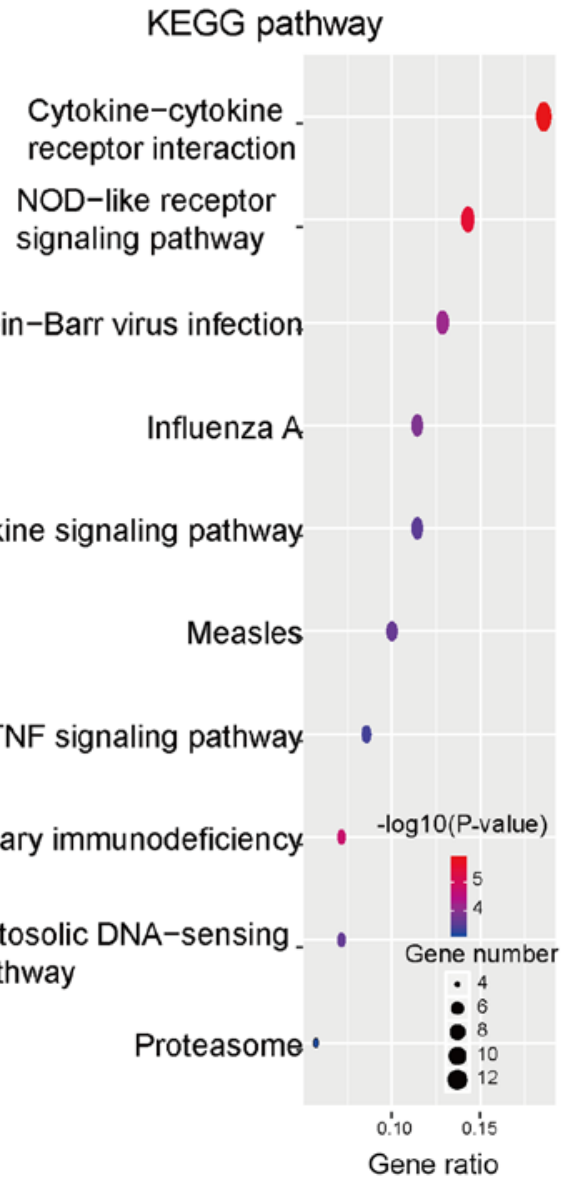

B

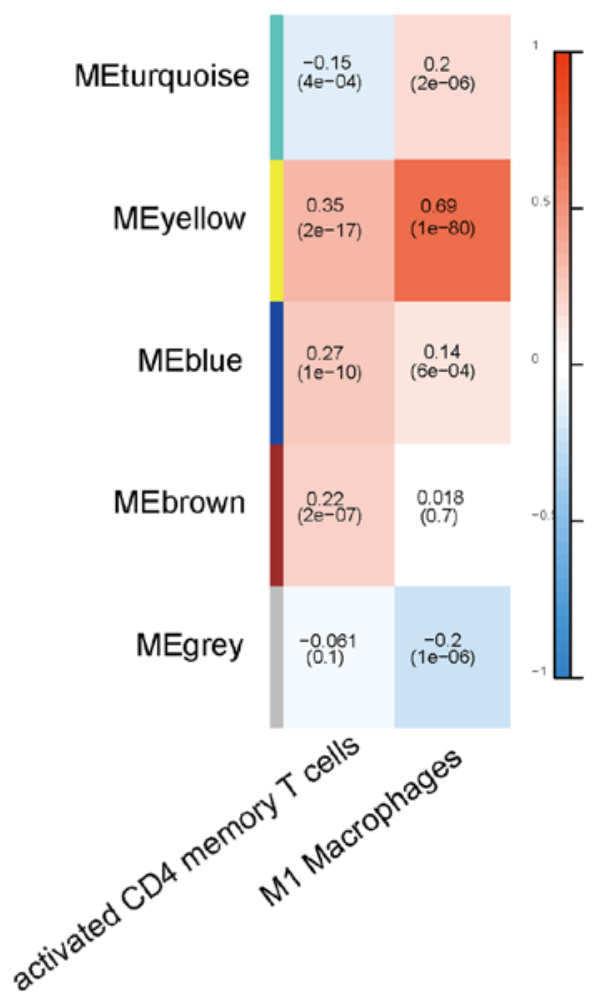

GO BP

regulation of innate immune response

response to virus.

T cell activation.

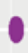

defense response to virus.

regulation of $\mathrm{T}$ cell activation-

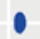

response to interferon-gamma.

cellular response to . interferon-gamma

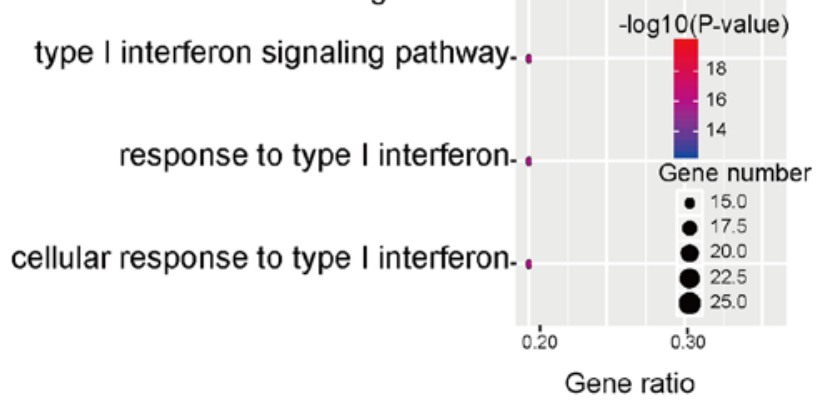

Figure 2. Immune cell infiltration associated modules identified in CRC. (A) Gene clustering and module identification by WGCNA analysis based on the recurrence-associated genes from GSE39582. The gene modules were signified by different colors and the grey modules indicated the genes that were unable to be merged. (B) Heat map of the correlation between modules and the ratio of M1 macrophages and activated CD4 ${ }^{+}$memory T cells. (C) KEGG and (D) GO BP analysis of the yellow module identified by WGCNA. The size and the color intensity of a circle represent the gene number and -log10 (P value), respectively. CRC, colorectal cancer; WGCNA, weighted correlation network analysis; KEGG, Kyoto Encyclopedia of Genes and Genomes; GO, gene ontology; BP, biological process. 
A

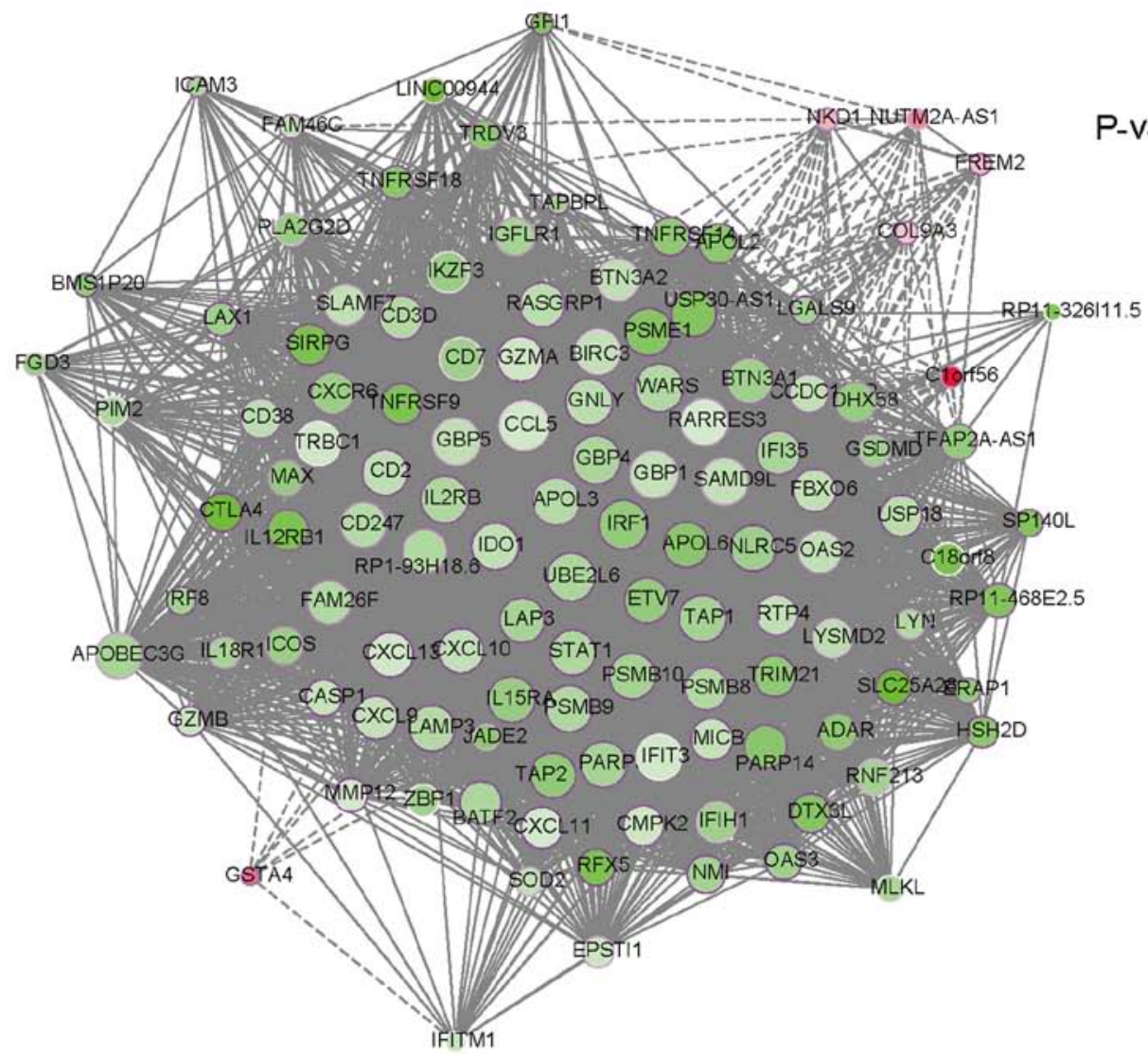

HR

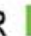
1

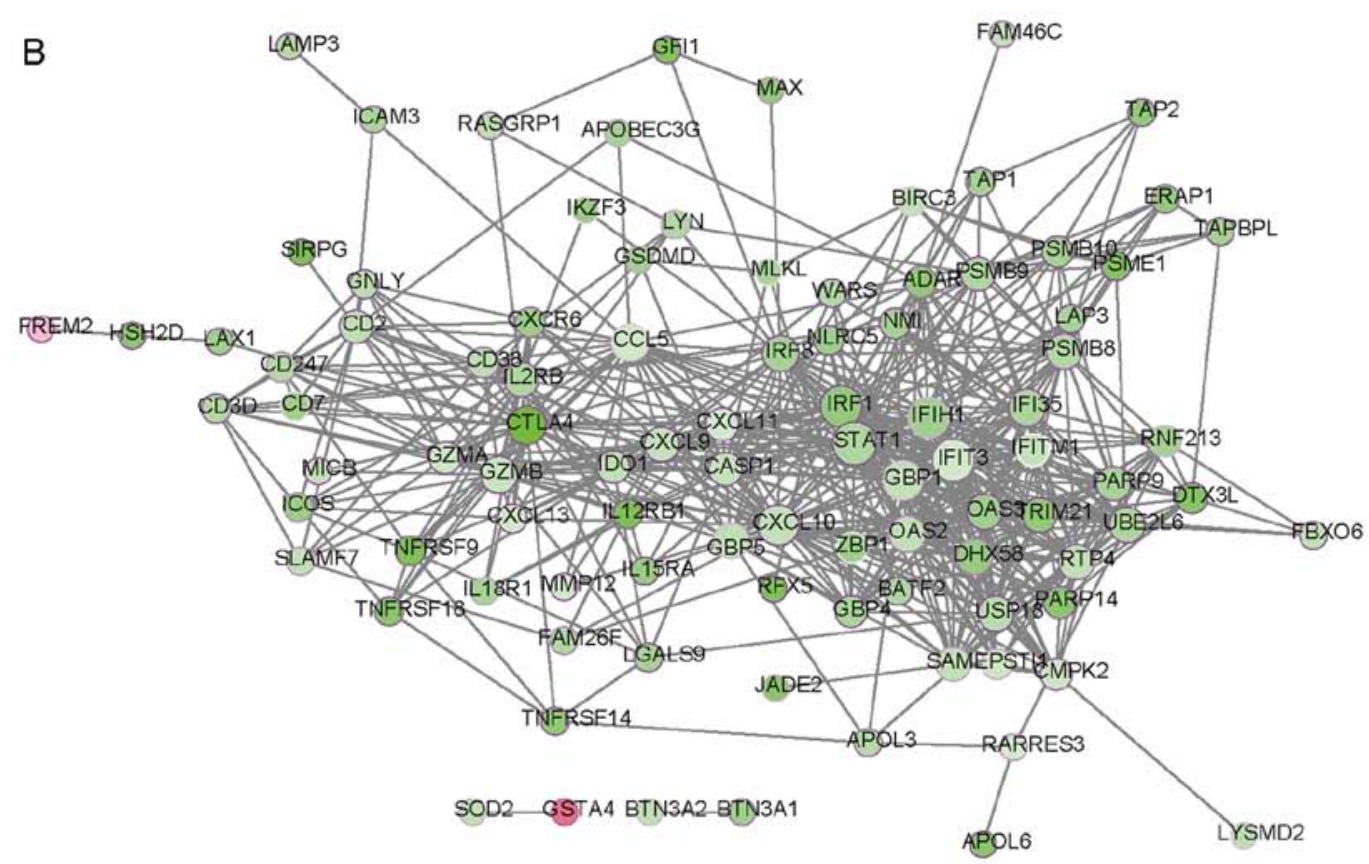

Figure 3. Co-expression network and PPI network from the yellow module. The construction of the (A) co-expression network and the (B) PPI network for genes in the yellow module. The filled color indicates the HRs of each gene and the border color reflects the P-value of each gene. The size of genes indicated the degree, which was able to reflect the number of links between the gene and others. Solid lines represent a positive correlation and dotted lines represent a negative correlation. PPI, protein-protein interaction; HR, hazard ratio.

Pearson's correlation coefficient between genes and the ratio of immune cells (27). $\mathrm{P}<0.05$ was considered to indicate a statistically significant difference (28). Data are presented as median \pm quartile for boxplot.

\section{Results}

Influence of immune cell infiltration on the recurrence of patients with CRC. A previous study demonstrated that the 

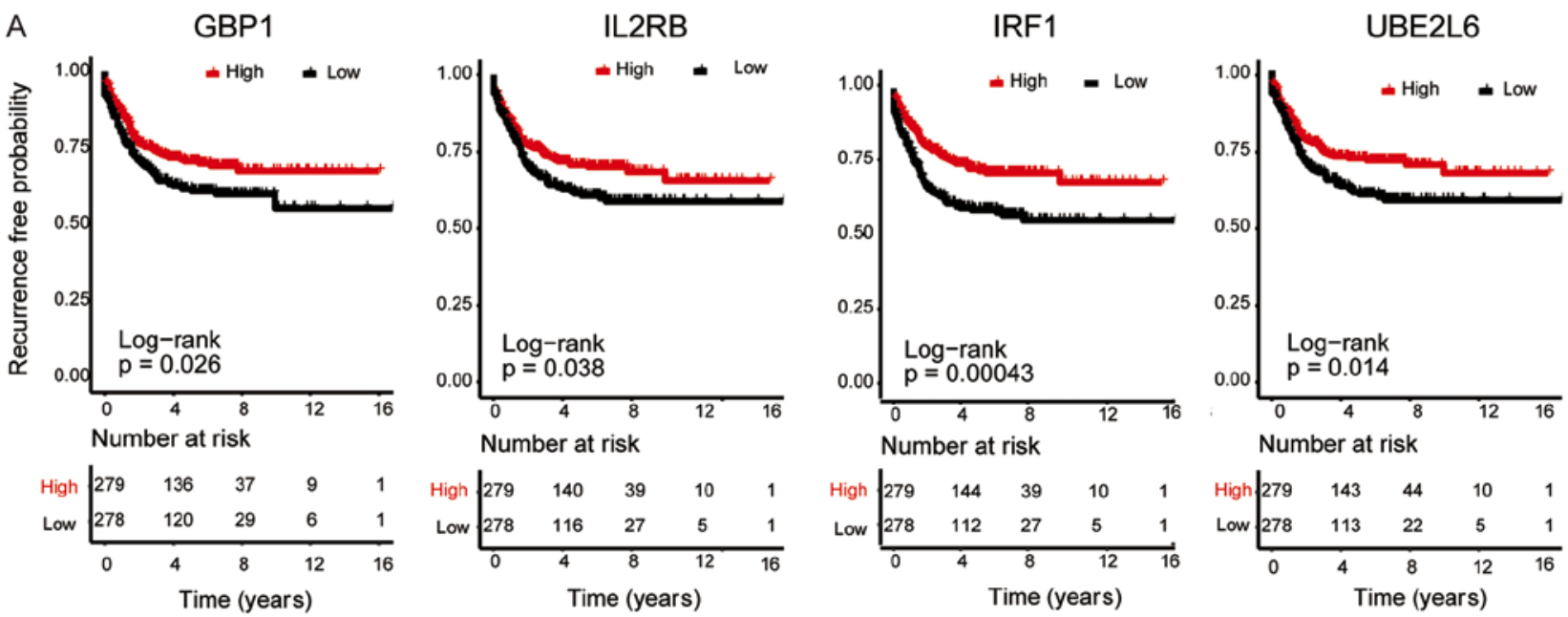

B

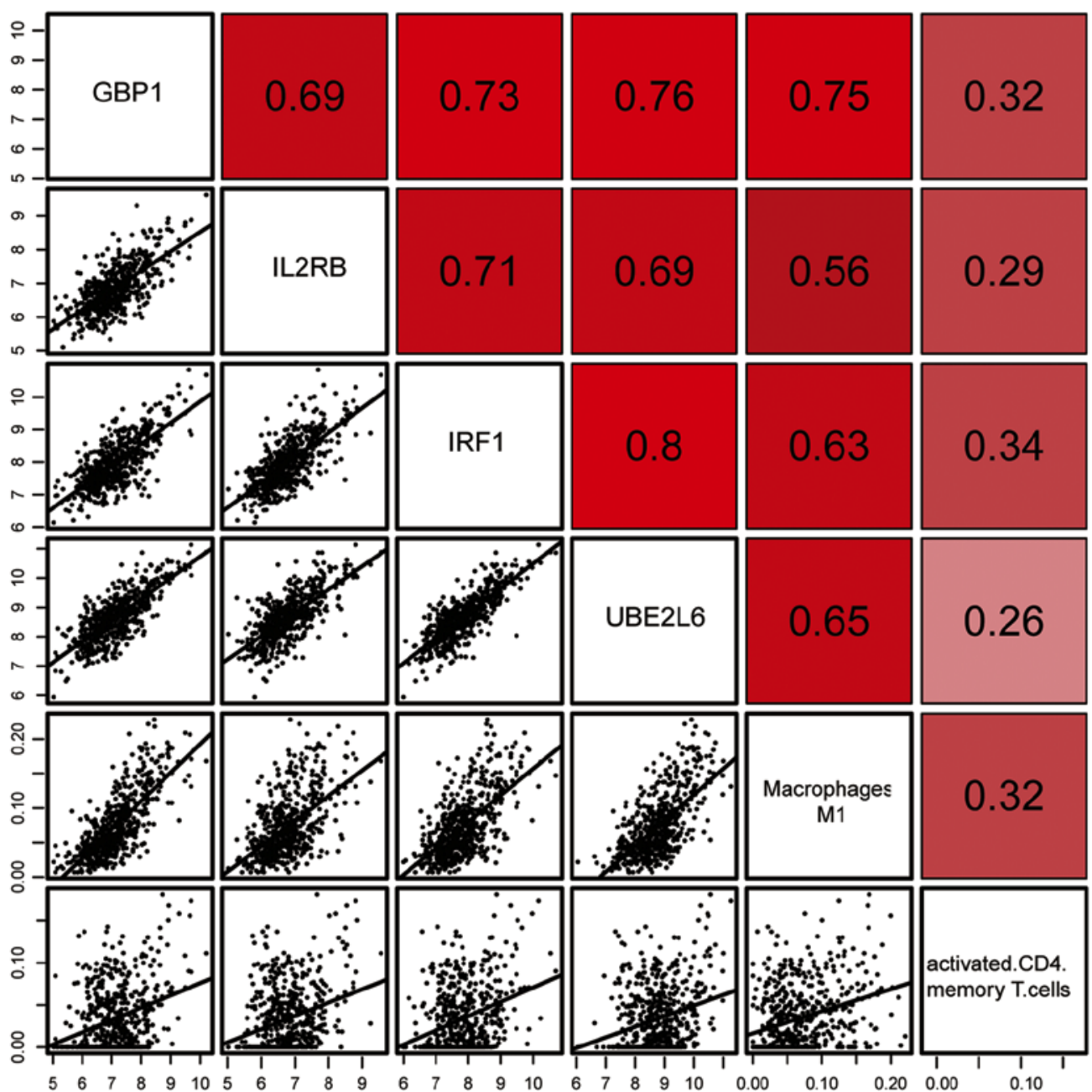

Figure 4. Kaplan Meier survival plots of RFS for hub genes in the yellow module and its correlation analysis with the ratio of M1 macrophages and ratio of activated CD4 memory T cells. (A) According to the median expression of each gene, the patients were stratified into a high-level group and a low-level group for Kaplan Meier survival plot analysis of RFS. (B) Association between the hub genes and the two recurrence-associated immune cells. RFS, recurrence free survival; GBP1, guanylate binding protein 1; IL2RB, interleukin 2 receptor subunit $\beta$; IRF1, interferon regulatory factor 1; UBE2L6, ubiquitin conjugating enzyme E2 L6.

mRNA expression of immune signature genes may predict the ratio of a number of immune cell subsets (15). To investigate the function of each TME immune cell subset infiltration in recurrence, CIBERSORT was used to calculate the ratio of 22 immune cell subsets in TME, for each patient with CRC, based on the mRNA expression data of immune signature genes. M1 macrophages and activated memory $\mathrm{CD}^{+} \mathrm{T}$ cells were indicated to be protective factors and indicative of improved RFS time ( $\mathrm{P}<0.01$; Fig. 1A and B). Due to the LM22, the molecular pattern of M1 macrophages identification 
Table I. Univariate Cox analyses and multivariate Cox analysis of 4 hub genes.

\begin{tabular}{|c|c|c|c|c|c|c|}
\hline \multirow[b]{2}{*}{ Gene } & \multicolumn{3}{|c|}{ Univariate Cox analysis } & \multicolumn{3}{|c|}{ Multivariate Cox analysis } \\
\hline & $95 \% \mathrm{CI}$ & P-value & HR & $95 \% \mathrm{CI}$ & P-value & $\mathrm{HR}$ \\
\hline GBP1 & 0.821 & $0.683-0.987$ & $0.035^{\mathrm{a}}$ & & & $>0.05$ \\
\hline IL2RB & 0.741 & $0.59-0.93$ & $0.01^{\mathrm{a}}$ & & & $>0.05$ \\
\hline UBE2L6 & 0.73 & $0.604-0.881$ & $0.001^{\mathrm{a}}$ & & & $>0.05$ \\
\hline IRF1 & 0.618 & $0.497-0.769$ & $<0.001^{\mathrm{a}}$ & 0.527 & $0.359-0.772$ & $0.001^{\mathrm{a}}$ \\
\hline
\end{tabular}

$\mathrm{HR}$, hazard ratio; CI, confidence interval; GBP1, guanylate binding protein 1; IL2RB, interleukin 2 receptor subunit $\beta$; IRF1, interferon regulatory factor 1; UBE2L6, ubiquitin conjugating enzyme E2 L6. ${ }^{\mathrm{P}} \mathrm{P}<0.05$.

included ADAMDEC1, AIF1, ALOX15, CCL13, CCL14, CCL18, CCL23, CCL8, CD209, CD4, CD68, CFP, CHI3L1, CLEC10A, CLEC4A, CLIC2, CRYBB1, EBI3, FAM198B, FES, FRMD4A, FZD2, GGT5, GSTT1, HRH1, HTR2B, MS4A6A, NME8, NPL, P2RY13, PDCD1LG2, RENBP, SIGLEC1, SLC15A3, TLR8, TREM2 and WNT5B. The molecular pattern of activated memory $\mathrm{CD} 4^{+} \mathrm{T}$ cells included CCL20, CD2, CD247, CD28, CD3D, CD3G, CD40LG, CD6, CD7, CDC25A, CSF2, CTLA4, CXCL13, DPP4, GPR171, GPR19, GZMB, ICOS, IFNG, IL12RB2, IL17A, IL26, IL2RA, IL3, IL4, IL9, LAG3, LCK, LTA, NKG7, ORC1, PMCH, RRP9, SH2D1A, SKA1, TNFRSF4, TNIP3, TRAC, TRAT1 and UBASH3A. The ratio of M1 macrophages and activated $\mathrm{CD}^{+}$memory $\mathrm{T}$ cells was significantly lower in patients with recurrence compared with patients with no recurrence $(\mathrm{P}<0.001$; Fig. 1C). Further analysis revealed that the ratio of $\mathrm{M} 1$ macrophages and activated $\mathrm{CD} 4^{+}$memory $\mathrm{T}$ cells demonstrated no significant difference between patients with a TP53 mutation and wild-type (WT) patients, but was significantly reduced in patients with the K-ras mutation compared with WT patients $(\mathrm{P}<0.01$; Fig. 1D and E). Additionally, the ratio of M1 macrophages and activated $\mathrm{CD}^{+}$memory $\mathrm{T}$ cells was significantly increased in patients with the B-Raf proto-oncogene, serine/threonine kinase mutation compared with WT patients $(\mathrm{P}<0.001$; Fig. $1 \mathrm{~F})$. In comparison with patients with advanced CRC, early-stage patients exhibited a significantly higher ratio of M1 macrophages and activated memory $\mathrm{CD} 4^{+} \mathrm{T}$ cells $(\mathrm{P}<0.05$; Fig. $1 \mathrm{G})$. These results revealed that the ratio of M1 macrophages and activated memory $\mathrm{CD} 4^{+} \mathrm{T}$ cells in TME serve a vital function in the recurrence of patients with CRC.

Identification of key modules associated with immune cell infiltration. To investigate the factors that influenced the ratio of M1 macrophages and activated memory $\mathrm{CD}^{+} \mathrm{T}$ cells, and the response of recurrence in patients with CRC, 3,530 recurrence-associated genes were identified using univariate Cox analysis, which may be used as independent factors for CRC. Subsequently, based on the mRNA expression of recurrence-associated genes, a co-expression network was constructed and key modules were identified using WGCNA, which were used to aggregate genes with the same expression pattern. The results revealed that the recurrence-associated genes may be grouped into 4 major modules, which were identified as blue, brown, turquoise and yellow modules (Fig. 2A). The association between the infiltration of every immune cell subset and the modules was then assessed. The data revealed that the yellow module exhibited a positive correlation with the ratio of M1 macrophages and the ratio of activated memory $\mathrm{CD} 4^{+} \mathrm{T}$ cells (Fig. 2B). KEGG pathway and GO enrichment analysis were then performed for the yellow module. KEGG pathway enrichment analysis revealed that influenza A, Epstein-Bar virus infection, NOD-like receptor signaling pathway and cytokine-cytokine receptor interaction were enriched in the yellow module (Fig. 2C). The results of the GO enrichment analysis revealed that the yellow module was mainly enriched in the cellular response to type I interferon, regulation of innate immune response, $\mathrm{T}$ cell activation, defense response to virus and response to virus (Fig. 2D). These data indicated that recurrence-associated genes enriched in the yellow module may be involved in regulating the infiltration or function of immune cells in TME.

Identified hub genes in the yellow module influenced the RFS time of patients with CRC. Hub genes, which exhibited the highest degree and greatest number of associations with other genes, serve a key function in the yellow module (29). To further investigate the biological functions of the yellow module, co-expression networks that were based on the WGCNA and PPI network were constructed to identify hub genes in the yellow module (Fig. 3A and B; Table SI). By ranking the degree of these networks, IRF1, C-C motif chemokine ligand 5 (CCL5), ubiquitin conjugating enzyme E2 L6 (UBE2L6), guanylate binding protein 1 (GBP1) and interleukin 2 receptor subunit beta (IL2RB) were identified as the hub genes in the yellow module. Kaplan-Meier curve and Log-rank analysis revealed that the expression of IRF1, UBE2L6, GBP1 and IL2RB significantly influenced the RFS time of patients with CRC $(\mathrm{P}<0.05$; Fig. 4A). All 4 hub genes indicated a significant positive correlation with each other, in addition to the ratio of M1 macrophages and activated memory $\mathrm{CD}^{+} \mathrm{T}$ cells (Fig. 4B).

IRF1 was able to predict the RFS time of patients with CRC. Although all hub genes exhibited the potential to be independent diagnostic biomarkers for the RFS time of patients with CRC, IRF1 most significantly influenced this and was indicated to be a potential diagnostic biomarker for RFS in 

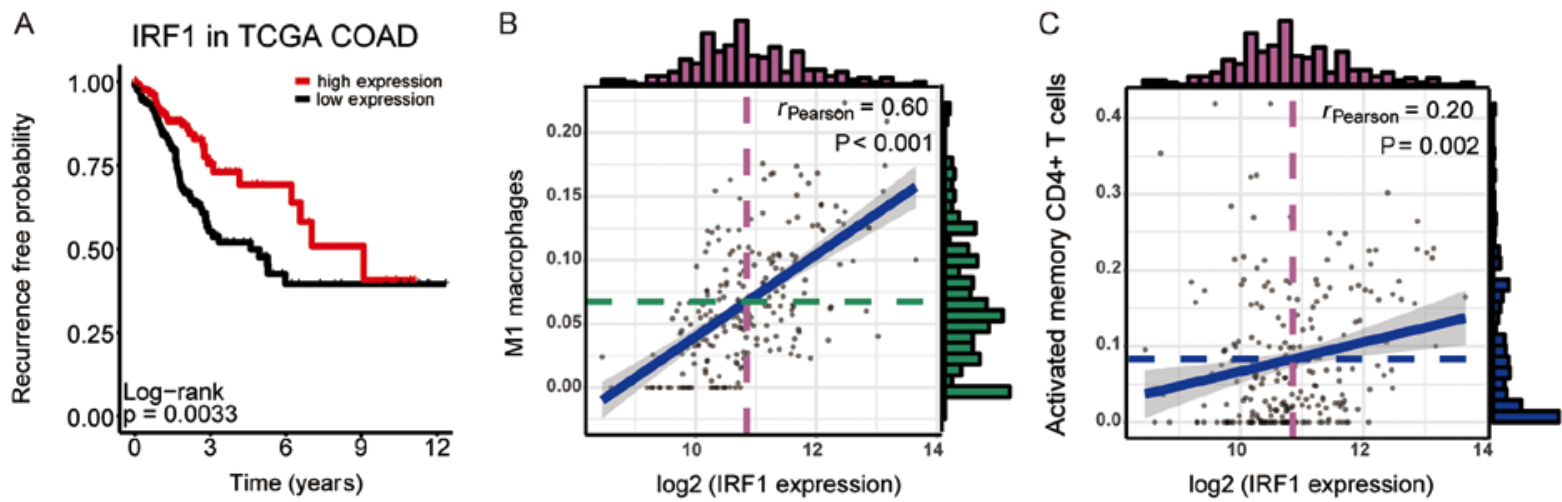

D GSEA based on GSE39582

E GSEA based on TCGA

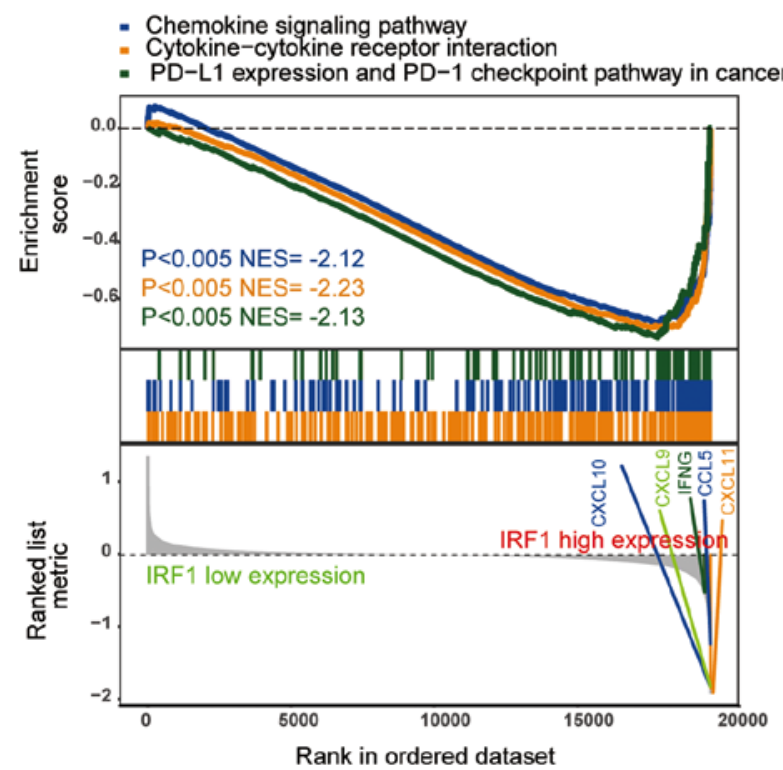

- Chemokine signaling pathway

- Cytokine-cytokine receptor interaction

- PD-L1 expression and PD-1 checkpoint pathway in cancer
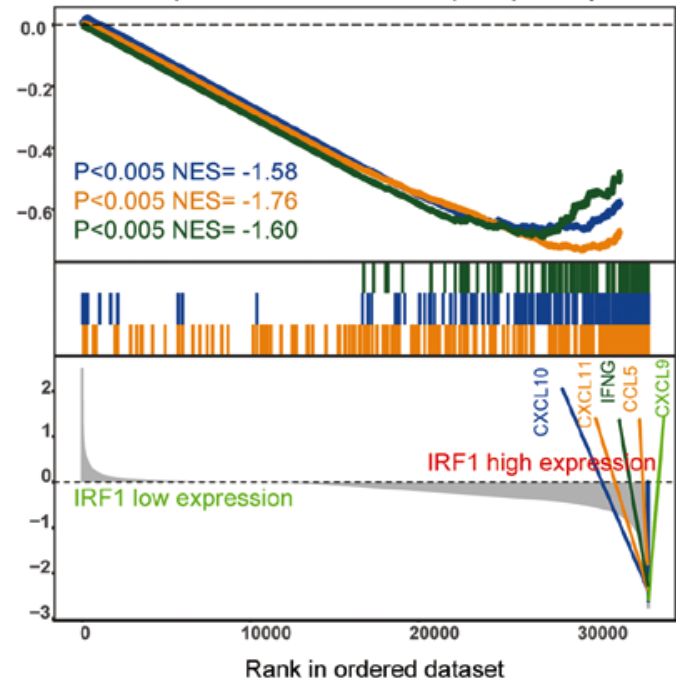

Figure 5. Verification of IRF1 prognostic signature using TCGA datasets and the GSEA of the DEGs between the high- and low-expression IRF1 groups. (A) Kaplan Meier survival plots of the association between recurrence-free survival and IRF1 expression in TCGA datasets. The association between IRF1 expression and the ratio of (B) M1 macrophages and (C) activated CD4+ memory T cells in TCGA datasets. The histogram exhibited the frequency in the value. The violet dotted line represents the median value of $\log _{2}$ (IRF1 expression) and the green dotted line represents the median value of the ratio of (B) M1 macrophages or (C) activated CD4 $4^{+}$memory T cells. The blue line revealed the line of best fit of the association between the $\log ^{2}$ (IRF1 expression) and cell ratio of (B) M1 macrophages or (C) activated CD4 $4^{+}$memory T cells. GSEA of the DEGs between the high- and low-expression IRF1 groups using (D) GSE39582 and (E) TCGA datasets. The P-value and NES of different pathways were indicated by different colors, respectively. IRF1, interferon regulatory factor 1; TCGA, The Cancer Genome Atlas; GSEA, Gene set enrichment analysis; PD-1, programmed cell death 1; PD-L1, programmed death ligand 1; CXCL, C-X-C motif chemokine ligand; IFNG, interferon $\gamma$; CCL5, C-C motif chemokine ligand 5; DEG, differentially expressed gene; NES, normalized enrichment score; COAD, colon adenocarcinoma.

patients with CRC $(\mathrm{P}=0.001$; Table I). TCGA data was then used for further validation, and the results revealed that patients with CRC and a lower expression of IRF1 exhibited a worse prognosis compared with those with a higher expression of IRF1, which is consistent with the results of the GSE39582 dataset $(\mathrm{P}<0.01$; Fig. 5A). Based on TCGA data, IRF1 expression was also significantly positively correlated with the ratio of $\mathrm{M} 1$ macrophages and activated memory $\mathrm{CD}^{+} \mathrm{T}$ cells $(\mathrm{P}<0.01$; Fig. 5B and C). An additional dataset from a Chinese cohort was also used, which contained the mRNA expression profiles of 52 human biopsy samples of CRC, and included overall survival time information (12). The data revealed that IRF1 expression in Chinese patients with CRC exhibited similar results compared with those identified in the French CRC cohort. Overall survival analysis indicated that Chinese patients with CRC and high IRF1 expression exhibited a high survival probability, although this was not significant. Patients with CRC and higher IRF1 expression exhibited better survival time compared with patients with lower IRF1 expression, and IRF1 expression demonstrated a positive association with the ratio of $\mathrm{M} 1$ macrophages and activated memory $\mathrm{CD} 4^{+} \mathrm{T}$ cells, though this was not statistically significant as it was a smaller sample size (Fig. S1). Although age exhibited a significant difference, there was no difference of all other clinical variables between these two cohorts, including sex, TNM stage, overall survival time and survival state (Table SII). This indicated that the expression of IRF1 may predict the RFS of patients with CRC in different cohorts, including Europeans, Americans and Asians.

Patients from GSE39582 and TCGA were divided into two groups according to the median IRF1 expression. GSEA was used to investigate the different KEGG pathways 
A
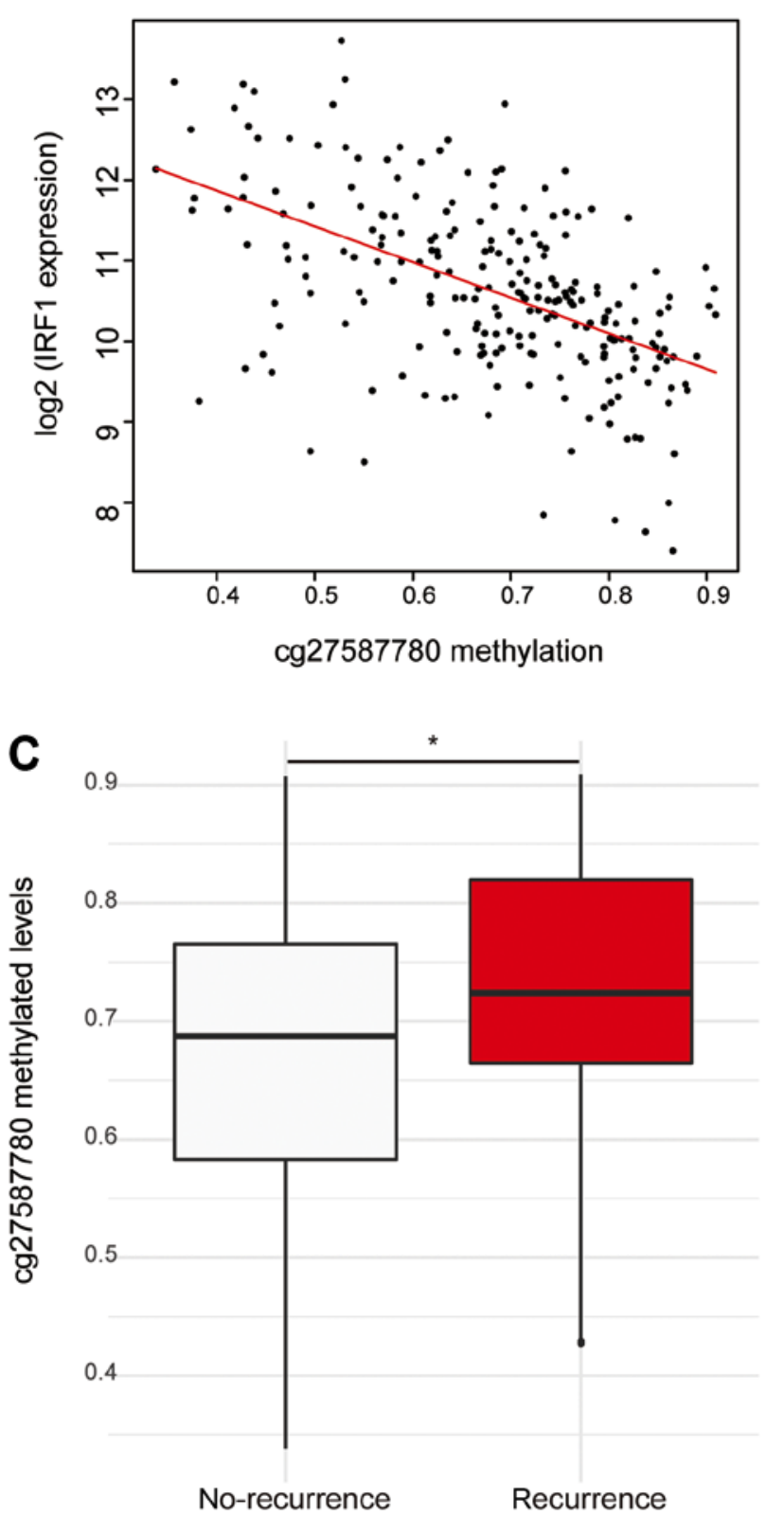

B
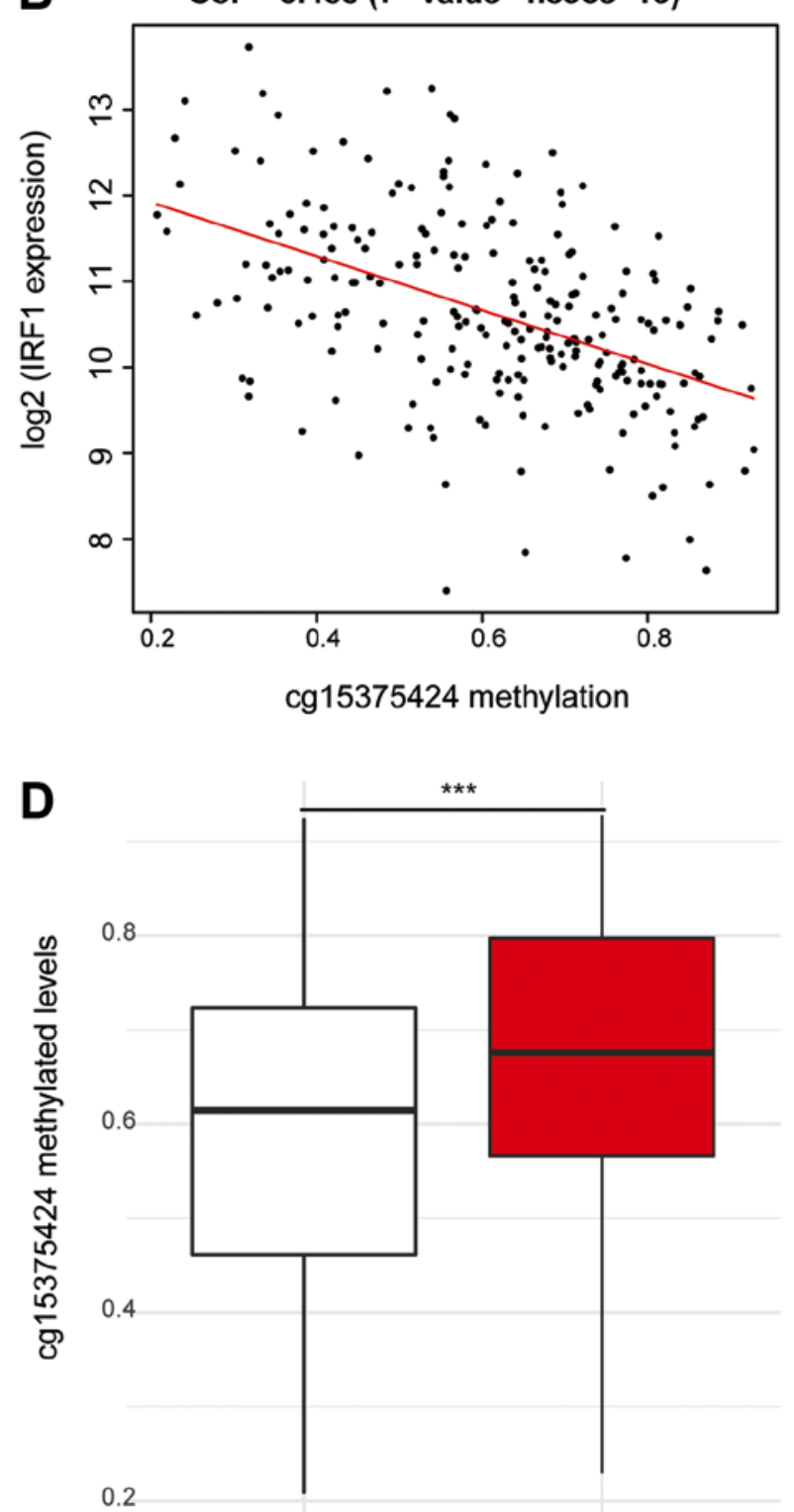

No-recurrence
Recurrence

Figure 6. Relevance of IRF1-associated methylated CpG sites to IRF1 expression and patient CRC recurrence. Correlation diagrams of IRF1 expression and the degree of DNA methylation of (A) cg27587780 and (B) cg15375424. A boxplot exhibited the degree of DNA methylation of (C) cg27587780 and (D) cg15375424 in patients with no-recurrence and recurrence. ${ }^{*} \mathrm{P}<0.05$ and ${ }^{* * *} \mathrm{P}<0.001$ (t-test), with comparisons shown by lines. The vertical axes demonstrate the lower quartile and upper quartile of the methylation level of the indicated site for no-recurrence (white) or recurrence (red) cohorts; and the line inside each box indicates the median of the methylation of the indicated sites in each group. IRF1, interferon regulatory factor 1; CRC, colorectal cancer.

between these groups, and the results demonstrated that pathways associated with immune cell recruitment and activation, including the chemokine signaling pathway, cytokine-cytokine receptor interaction and programmed death ligand 1 expression and programmed cell death 1 checkpoint pathways in cancer, were upregulated in the IRF1 high-expression group. A number of chemokines, including C-X-C motif chemokine ligand (CXCL) 9-11 and CCL5, were highly expressed in the IRF1 high-expression group. These results indicated that a higher expression of IRF may reduce the risk of recurrence through influencing the recruitment and activation of immune cells, particularly M1 macrophages and activated memory $\mathrm{CD} 4^{+} \mathrm{T}$ cells.
DNA methylation levels of two CpG sites (cg27587780 and cg15375424) are negatively correlated with the expression of IRF1 and positively correlated with the recurrence of patients with CRC. To investigate the aberrant expression of IRF1, the association between the degree of DNA methylation of IRF1-associated methylated $\mathrm{CpG}$ sites and the expression of IRF1 were analyzed based on TCGA dataset. The results indicated that two CpG sites, cg27587780 and $\operatorname{cg} 15375424$, were significant negatively correlated with IRF1 expression $(\mathrm{P}<0.001$; Fig. $6 \mathrm{~A}$ and $\mathrm{B})$. These two methylated $\mathrm{CpG}$ sites were hypermethylated in patients with $\mathrm{CRC}$ recurrence compared with patients with CRC without recurrence $(\mathrm{P}<0.05$; Fig. 6C and D). 


\section{Discussion}

Colorectal cancer is the third leading cause of cancer-associated mortality globally $(1,2)$. Although the majority of patients with CRC are treated using surgery combined with chemotherapy, local recurrence and remote metastases following therapy often influence their survival rate (30). At present, there is a lack of effective biomarkers for CRC recurrence. TME, particularly immune cell infiltration, serves an important function in affecting the metastasis and recurrence of patients with CRC (31). Therefore, investigating the mechanisms of TME and TME-associated factors may be useful to identify novel biomarkers for CRC prognosis and may provide more effective, target-specific or personalized therapeutic strategies for patients with CRC.

In the present study, based on the data from GSE39582, M1 macrophages and activated memory $\mathrm{CD}^{+} \mathrm{T}$ cells were indicated to be protective factors for the survival of patients with CRC. Patients with a high score of M1 macrophages and activated memory $\mathrm{CD} 4^{+} \mathrm{T}$ cells exhibited a lower risk of recurrence. $\mathrm{M} 1$ macrophages and activated memory $\mathrm{CD} 4^{+} \mathrm{T}$ cells have been confirmed as tumor-preventing cells in CRC, but factors affecting the distribution of these cells in TME remain unclear $(32,33)$. The results of the present study revealed that a module, which contained 120 genes from recurrence-associated genes in CRC, was positively correlated with these two immune cell subsets in TME. In this module, the top 5 hub genes were identified to be IRF1, CCL5, UBE2L6, GBP1 and IL2RB.

Further analysis using a Kaplan-Meier curve and Log-rank test revealed that all top 5 hub genes, except CCL5, influenced the RFS of patients with CRC. IRF1, which belongs to the IRF family (34), is weakly expressed in resting dendritic cells and macrophages, but is induced by interferon- $\gamma($ IFN- $\gamma)$ in M1 polarized macrophages $(35,36)$. Previous studies have revealed that IRF1 inhibited the proliferation and metastasis of CRC $(37,38)$. UBE2L6, which is also known as UBCH8, promotes apoptosis in cervical cancer cells (39). In the present study, the low expression of UBE2L6 was associated with a poor prognosis in patients with CRC. GBP-1 is highly induced by IFN- $\gamma$ in a number of different cell types, and functions as a tumor suppressor, which arrests tumor evasion in CRC $(40,41)$. IL2RB, which is a receptor on numerous different effector immune cells of interleukin-2, promotes antitumor immunity (42). All aforementioned reported results are consistent with the results of the present study, which indicated that patients with CRC and a higher expression of IRF1, UBE2L6, GBP1 or IL2RB exhibited better RFS time.

Multivariate Cox analysis revealed that IRF1 may be a diagnostic biomarker for RFS in patients with CRC among these genes, which was also validated using TCGA datasets and a Chinese cohort. In aggressive neuroblastoma, a previous study revealed that IRF1 and nuclear factor- $\mathrm{\kappa B}$ restored MHC class I-restricted tumor antigen processing and presentation to cytotoxic T cells (43). A tumor-derived exosome, which was induced by IRF-1 overexpression, enhanced the anti-tumor immune response (44). IRF1 expression in tumor cells was also reported to be critical for the immune response to adoptive T cell therapy (45) and the antitumor immunity of cyclophosphamide (46). Consistently, the results of the present study revealed that IRF1 expression was correlated with the expression of CXCL9, CXCL10, CXCL11 and IFNG, which have been demonstrated to influence macrophage infiltration and memory $\mathrm{CD}^{+} \mathrm{T}$ cell activation $(47,48)$. The increased predictive ability of IRF1 mRNA expression may be due to the fact IRF1 is able to regulate immune subsets infiltration into the TME of CRC. Epigenetic gene silencing that is caused by DNA methylation has been widely accepted as a major mechanism of tumor recurrence (49). IRF4, IRF5 and IRF8 have been reported to be frequently suppressed in gastric cancer due to DNA methylation (50). In the present study, two $\mathrm{CpG}$ sites, cg27587780 and cg15375424, in the IRF1 DNA region, were demonstrated to be significantly negatively correlated with IRF1 expression. This result may explain the abnormal IRF1 expression.

Collectively, the expression of IRF1 may predict the RFS of patients with CRC in different cohorts, which may be due to IRF1 serving a function in regulating the ratio of M1 macrophages and activated memory $\mathrm{CD} 4^{+} \mathrm{T}$ cells. This result may be considered useful information for use in treatment or immunotherapy in clinical practice.

\section{Acknowledgements}

Not applicable.

\section{Funding}

The present study was supported by the National Natural Science Foundation of China (grant. no. 81372272) and the Provincial Natural Science Foundation of Tibet (grant. no. XZ2019ZRG-135).

\section{Availability of data and materials}

The datasets used and/or analyzed during the current study are available from the corresponding author on reasonable request.

\section{Authors' contributions}

YW devised the concept, designed experiments, analyzed data, and wrote the manuscript; SZ analyzed data; JY devised the concept, designed the research, supervised the study, and revised the paper. All authors read and approved the final manuscript.

\section{Ethics approval and consent to participate}

This research abides by the International and National regulations in accordance with the Declaration of Helsinki. It was approved by the Ethics Committee of the Fourth People's Hospital of Shaanxi (Shanxi, China).

\section{Patient consent for publication}

Not applicable.

\section{Competing interests}

The authors have declared that they have no competing interests. 


\section{References}

1. Chen W, Zheng R, Baade PD, Zhang S, Zeng H, Bray F, Jemal A, Yu XQ and He J: Cancer statistics in China, 2015. CA Cancer J Clin 66: 115-132, 2016.

2. Arnold M, Sierra MS, Laversanne M, Soerjomataram I, Jemal A and Bray F: Global patterns and trends in colorectal cancer incidence and mortality. Gut 66: 683-691, 2017.

3. Baca B, Beart RW Jr and Etzioni DA: Surveillance after colorectal cancer resection: A systematic review. Dis Colon Rectum 54: 1036-1048, 2011.

4. Inadomi JM: Screening for colorectal neoplasia. N Engl J Med 376: 1599-1600, 2017.

5. Lee JS: Exploring cancer genomic data from the cancer genome atlas project. BMB Rep 49: 607-611, 2016.

6. Yip S, Christofides A, Banerji S, Downes MR, Izevbaye I, Lo B, MacMillan A, McCuaig J, Stockley T, Yousef GM and Spatz A: A Canadian guideline on the use of next-generation sequencing in oncology. Curr Oncol 26: e241-e254, 2019.

7. Sun D, Chen J, Liu L, Zhao G, Dong P, Wu B, Wang J and Dong L: Establishment of a 12-gene expression signature to predict colon cancer prognosis. PeerJ 6: e4942, 2018.

8. Lee JH, Jung S, Park WS, Choe EK, Kim E, Shin R, Heo SC Lee JH, Kim K and Chai YJ: Prognostic nomogram of hypoxia-related genes predicting overall survival of colorectal cancer-analysis of TCGA database. Sci Rep 9: 1803, 2019.

9. Yan X, Jiao SC, Zhang GQ, Guan Y and Wang JL: Tumor-associated immune factors are associated with recurrence and metastasis in non-small cell lung cancer. Cancer Gene Ther 24: 57-63, 2017.

10. Edgar R, Domrachev M and Lash AE: Gene expression omnibus: NCBI gene expression and hybridization array data repository. Nucleic Acids Res 30: 207-210, 2002

11. Marisa L, de Reynies A, Duval A, Selves J, Gaub MP, Vescovo L, Etienne-Grimaldi MC, Schiappa R, Guenot D, Ayadi M, et al: Gene expression classification of colon cancer into molecular subtypes: Characterization, validation, and prognostic value. PLoS Med 10: e1001453, 2013.

12. An N, Shi X, Zhang Y, Lv N, Feng L, Di X, Han N, Wang G, Cheng $S$ and Zhang K: Discovery of a novel immune gene signature with profound prognostic value in colorectal cancer: A model of cooperativity disorientation created in the process from development to cancer. PLoS One 10: e0137171, 2015.

13. Mounir M, Lucchetta M, Silva TC, Olsen C, Bontempi G, Chen X, Noushmehr H, Colaprico A and Papaleo E: New functionalities in the TCGAbiolinks package for the study and integration of cancer data from GDC and GTEx. PLoS Comput Biol 15: e1006701, 2019.

14. Newman AM, Liu CL, Green MR, Gentles AJ, Feng W, Xu Y, Hoang CD, Diehn M and Alizadeh AA: Robust enumeration of cell subsets from tissue expression profiles. Nat Methods 12: 453-457, 2015

15. Chen B, Khodadoust MS, Liu CL, Newman AM and Alizadeh AA: Profiling tumor infiltrating immune cells with CIBERSORT. Methods Mol Biol 1711: 243-259, 2018.

16. Li Q, Su YL and Shen WX: A novel prognostic signature of seven genes for the prediction in patients with thymoma. J Cancer Res Clin Oncol 145: 109-116, 2019.

17. Yang Y, Lu Q, Shao X, Mo B, Nie X, Liu W, Chen X, Tang Y, Deng $Y$ and Yan J: Development of A three-gene prognostic signature for hepatitis B virus associated hepatocellular carcinoma based on integrated transcriptomic analysis. J Cancer 9: 1989-2002, 2018.

18. Langfelder P and Horvath S: WGCNA: An R package for weighted correlation network analysis. BMC Bioinformatics 9: $559,2008$.

19. Yuan L, Zeng G, Chen L, Wang G, Wang X, Cao X, Lu M, Liu X, Qian G, Xiao Y and Wang X: Identification of key genes and pathways in human clear cell renal cell carcinoma (ccRCC) by co-expression analysis. Int J Biol Sci 14: 266-279, 2018.

20. Szklarczyk D, Gable AL, Lyon D, Junge A, Wyder S, Huerta-Cepas J, Simonovic M, Doncheva NT, Morris JH, Bork P, et al: STRING v11: Protein-protein association networks with increased coverage, supporting functional discovery in genome-wide experimental datasets. Nucleic Acids Res 47(D1): D607-D613, 2019.

21. Yu G, Wang LG, Han Y and He QY: ClusterProfiler: An R package for comparing biological themes among gene clusters. OMICS 16: 284-287, 2012
22. Zhu Z, Jin Z, Deng Y, Wei L, Yuan X, Zhang M and Sun D: Co-expression network analysis identifies four hub genes associated with prognosis in soft tissue sarcoma. Front Genet 10: 37, 2019.

23. Liu J, Zhou S, Li S, Jiang Y, Wan Y, Ma X and Cheng W: Eleven genes associated with progression and prognosis of endometrial cancer (EC) identified by comprehensive bioinformatics analysis. Cancer Cell Int 19: 136, 2019.

24. Gu S, Lin S, Ye D, Qian S, Jiang D, Zhang X, Li Q, Yang J, Ying X, Li Z, et al: Genome-wide methylation profiling identified novel differentially hypermethylated biomarker MPPED2 in colorectal cancer. Clin Epigenetics 11: 41, 2019.

25. Turcan S, Rohle D, Goenka A, Walsh LA, Fang F, Yilmaz E, Campos C, Fabius AW, Lu C, Ward PS, et al: IDH1 mutation is sufficient to establish the glioma hypermethylator phenotype. Nature 483: 479-483, 2012.

26. Sun X, Han Q, Luo H, Pan X, Ji Y, Yang Y, Chen H, Wang F, Lai W, Guan X, et al: Profiling analysis of long non-coding RNAs in early postnatal mouse hearts. Sci Rep 7: 43485, 2017.

27. Pesenti C, Navone SE, Guarnaccia L, Terrasi A, Costanza J, Silipigni R, Guarneri S, Fusco N, Fontana L, Locatelli M, et al: The genetic landscape of human glioblastoma and matched primary cancer stem cells reveals intratumour similarity and intertumour heterogeneity. Stem Cells Int 2019: 2617030 , 2019.

28. Wen Q, Yang Y, Chen XH, Pan XD, Han Q, Wang D, Deng YC, Li XH, Yan J and Zhou JH: Competing endogenous RNA screening based on long noncoding RNA-messenger RNA co-expression profile in Hepatitis B virus-associated hepatocarcinogenesis. J Tradit Chin Med 37: 510-521, 2017.

29. Chen P, Wang F, Feng J, Zhou R, Chang Y, Liu J and Zhao Q: Co-expression network analysis identified six hub genes in association with metastasis risk and prognosis in hepatocellular carcinoma. Oncotarget 8: 48948-48958, 2017.

30. Zhang Y, Chen $\mathrm{Z}$ and $\mathrm{Li} \mathrm{J}$ : The current status of treatment for colorectal cancer in China: A systematic review. Medicine (Baltimore) 96: e8242, 2017.

31. Tosolini M, Kirilovsky A, Mlecnik B, Fredriksen T, Mauger S, Bindea G, Berger A, Bruneval P, Fridman WH, Pages F and Galon J: Clinical impact of different classes of infiltrating T cytotoxic and helper cells (Th1, th2, treg, th17) in patients with colorectal cancer. Cancer Res 71: 1263-1271, 2011.

32. Deschoolmeester V, Baay M, Lardon F, Pauwels P and Peeters M: immune cells in colorectal cancer: Prognostic relevance and role of MSI. Cancer Microenviron 4: 377-392, 2011.

33. Edin S, Wikberg ML, Dahlin AM, Rutegard J, Oberg A, Oldenborg PA and Palmqvist R: The distribution of macrophages with a M1 or M2 phenotype in relation to prognosis and the molecular characteristics of colorectal cancer. PLoS One 7: e47045, 2012

34. Alsamman K and El-Masry OS: Interferon regulatory factor 1 inactivation in human cancer. Biosci Rep 38: pii: BSR20171672, 2018.

35. Xie C, Liu C, Wu B, Lin Y, Ma T, Xiong H, Wang Q, Li Z, Ma C and Tu Z: Effects of IRF1 and IFN- $\beta$ interaction on the M1 polarization of macrophages and its antitumor function. Int J Mol Med 38: 148-160, 2016.

36. Huang C, Lewis C, Borg NA, Canals M, Diep H, Drummond GR, Goode RJ, Schittenhelm RB, Vinh A, Zhu M, et al: Proteomic identification of interferon-induced proteins with tetratricopeptide repeats as markers of M1 macrophage polarization. J Proteome Res 17: 1485-1499, 2018.

37. Gunthner R and Anders HJ: Interferon-regulatory factors determine macrophage phenotype polarization. Mediators Inflamm 2013: 731023, 2013.

38. Hong M, Zhang Z, Chen Q, Lu Y, Zhang J, Lin C, Zhang F, Zhang W, Li X, Zhang W and Li X: IRF1 inhibits the proliferation and metastasis of colorectal cancer by suppressing the RAS-RAC1 pathway. Cancer Manag Res 11: 369-378, 2018.

39. Zhang Q, Qiao L, Wang X, Ding C and Chen JJ: UHRF1 epigenetically down-regulates $\mathrm{UbcH} 8$ to inhibit apoptosis in cervical cancer cells. Cell Cycle 17: 300-308, 2018.

40. Britzen-Laurent N, Lipnik K, Ocker M, Naschberger E, Schellerer VS, Croner RS, Vieth M, Waldner M, Steinberg P, Hohenadl C and Stürzl M: GBP-1 acts as a tumor suppressor in colorectal cancer cells. Carcinogenesis 34: 153-162, 2013.

41. Britzen-Laurent N, Herrmann C, Naschberger E, Croner RS and Sturzl M: Pathophysiological role of guanylate-binding proteins in gastrointestinal diseases. World J Gastroenterol 22: 6434-6443, 2016. 
42. Malek TR and Castro I: Interleukin-2 receptor signaling: At the interface between tolerance and immunity. Immunity 33 : $153-165,2010$

43. Lorenzi S, Forloni M, Cifaldi L, Antonucci C, Citti A, Boldrini R, Pezzullo M, Castellano A, Russo V, van der Bruggen P, et al: IRF1 and NF-kB restore MHC class I-restricted tumor antigen processing and presentation to cytotoxic $\mathrm{T}$ cells in aggressive neuroblastoma. PLoS One 7: e46928, 2012.

44. Yang MQ, Du Q, Varley PR, Goswami J, Liang Z, Wang R, Li H, Stolz DB and Geller DA: Interferon regulatory factor 1 priming of tumour-derived exosomes enhances the antitumour immune response. Br J Cancer 118: 62-71, 2018.

45. Cascone T, McKenzie JA, Mbofung RM, Punt S, Wang Z, Xu C Williams LJ, Wang Z, Bristow CA, Carugo A, et al: Increased tumor glycolysis characterizes immune resistance to adoptive $t$ cell therapy. Cell Metab 27: 977-987.e4, 2018.

46. Buccione C, Fragale A, Polverino F, Ziccheddu G, Arico E, Belardelli F, Proietti E, Battistini A and Moschella F: Role of interferon regulatory factor 1 in governing Treg depletion, Th1 polarization, inflammasome activation and antitumor efficacy of cyclophosphamide. Int J Cancer 142: 976-987, 2018.

47. Zhuang J, Shan Z, Ma T, Li C, Qiu S, Zhou X, Lin L and Qi Z: CXCL9 and CXCL10 accelerate acute transplant rejection mediated by alloreactive memory $\mathrm{T}$ cells in a mouse retransplantation model. Exp Ther Med 8: 237-242, 2014.
48. Corbera-Bellalta M,Planas-Rigol E,Lozano E, Terrades-García N, Alba MA, Prieto-González S, García-Martínez A, Albero R, Enjuanes A, Espígol-Frigolé G, et al: Blocking interferon $\gamma$ reduces expression of chemokines CXCL9, CXCL10 and CXCL11 and decreases macrophage infiltration in ex vivo cultured arteries from patients with giant cell arteritis. Ann Rheum Dis 75: 1177-1186, 2016.

49. Nassiri F, Mamatjan Y, Suppiah S, Badhiwala JH, Mansouri S, Karimi S, Saarela O, Poisson L, Gepfner-Tuma I, Schittenhelm J, et al: DNA methylation profiling to predict recurrence risk in meningioma: Development and validation of a nomogram to optimize clinical management. Neuro Oncol, Jan 3, 2019 (Epub ahead of print).

50. Yamashita M, Toyota M, Suzuki H, Nojima M, Yamamoto E, Kamimae S, Watanabe Y, Kai M, Akashi H, Maruyama R, et al: DNA methylation of interferon regulatory factors in gastric cancer and noncancerous gastric mucosae. Cancer Sci 101: 1708-1716, 2010. Attribution-NonCommercial-NoDerivatives 4.0 International (CC BY-NC-ND 4.0) License. 